\title{
Mitral valve replacement in children: mortality, morbidity, and haemodynamic status up to medium term follow up
}

\author{
C van Doorn, R Yates, V Tsang, $M$ deLeval, $M$ Elliott
}

\begin{abstract}
Objective-To investigate the outcome of mechanical mitral valve replacement in children after up to 11 years of follow up.

Design-Retrospective analysis of case records. Operative survivors underwent echocardiographic studies to define current haemodynamic status and prosthetic valve function.

Setting-Tertiary referral centre.

Patients-All 54 children who underwent mitral valve replacement between January 1987 and December 1997.

Results - 30 day mortality was $20.3 \%$ and was associated with small valve size and supra-annular position. The actuarial freedom from the following events at five years $(70 \%$ confidence interval (CI)) was: death, including 30 day mortality and transplantation, $68 \%$ (70\% CI $62 \%$ to $75 \%$ ); bleeding, $89 \%$ ( $70 \%$ CI $84 \%$ to $94 \%$ ); non-structural valve dysfunction and reoperation, $92 \%$ ( $70 \%$ CI $87 \%$ to $97 \%$ ). The incidence of endocarditis and thromboembolism was low and there was no structural valve failure. Event-free survival was $52 \%$ ( $70 \%$ CI $45 \%$ to $60 \%$ ). Low weight, young age, and small valve size increased the chance of death or reoperation. On echocardiography, left ventricular dilatation and wall motion abnormalities were often observed. A high mean gradient over the prosthesis was associated with small valve size but not with length of follow up.

Conclusions-With the use of mechanical prostheses for mitral valve replacement in children, the problem of structural valve failure is no longer an issue. However, the procedure is still associated with a high complication rate, both at surgery and during follow up, and should therefore be reserved for patients in whom valve repair is not technically feasible.

(Heart 2000;84:636-642)
\end{abstract}

Keywords: mitral valve replacement; prosthetic mitral valve; child; outcome

Congenital mitral valve defects are rare, comprise a wide spectrum of morphological abnormalities of the valve, and are often associated with other cardiovascular malformations. ${ }^{12}$ Patients often present early in life with heart failure which is refractory to medical treatment. Despite advances in the surgical treatment of complex congenital heart disease, ${ }^{3-5}$ mitral valve repair is not always possible, and replacement of the valve with a prosthesis may be the only option. Mitral valve replacement in childhood may also be necessary when a previously normal valve is severely damaged by endocarditis or other pathological processes.

Bioprostheses were initially the preferred valve substitute, primarily because anticoagulation could be avoided. However, in children the durability of these valves is severely limited by early calcification ${ }^{6}$ and therefore mechanical valves became the preferred option, albeit at the expense of life long anticoagulation. In contrast to the adult population, in which the incidence of bleeding, thromboembolism, and other prosthetic valve related complications is well documented, ${ }^{7}$ information on these events in children is scarce and often based on small series of prostheses which are no longer in general use. ${ }^{8-11}$ Moreover, unlike in adults, the fixed size prosthesis will ultimately become too small in the growing child, resulting in restric- tion of haemodynamic function and necessitating redo replacement with a larger size of valve. $^{12}$

In this paper we report on our recent experience with mitral valve replacement in children. We studied mortality and morbidity retrospectively in all the patients who underwent mitral valve replacement at our institution since 1987, and performed echocardiographic studies in operative survivors to define current haemodynamic status and prosthetic valve function. In addition, quality of life was investigated and is reported in a companion paper. ${ }^{13}$

\section{Methods}

STUDY POPULATION

We reviewed the case notes of all patients who underwent mitral valve replacement at Great Ormond Street Hospital for Children between January 1987 and December 1997. Hospital records before 1987 were not complete enough for reliable data entry. Patients with left atrial isomerism, atrioventricular discordance, and univentricular hearts were excluded. The study was approved by the medical ethics committee of our institution.

Fifty four patients ( 29 girls, 25 boys) underwent a total of 59 mitral valve replacements. This represents $15.1 \%$ (59/389) of systemic left atrioventricular valve operations in biventricular hearts performed at our institution 
Table 1 Diagnoses in patients who underwent mitral valve replacement

\begin{tabular}{ll}
\hline Diagnosis & $\begin{array}{l}\text { Number of } \\
\text { patients }\end{array}$ \\
\hline $\begin{array}{l}\text { Congenital heart disease } \\
\quad \text { Congenital mitral regurgitation (12) }\end{array}$ & 39 \\
$\quad$ Congenital mitral stenosis (10) & \\
AVSD (complete 12, partial 5) & \\
Endocarditis (bacterial 7, viral 1) & 8 \\
$\quad$ Normal mitral valve (4) & \\
$\quad$ Previous AVSD repair (2) & \\
Previous rheumatic fever (1) & \\
Marfan's syndrome (1) & \\
Rheumatic fever & \\
Prosthetic mitral stenosis (first mechanical valve & 5 \\
placed before 1987) & 5 \\
Original diagnosis congenital mitral stenosis \\
$\quad$ (4) \\
$\quad$ Original diagnosis viral endocarditis (1) \\
Total
\end{tabular}

AVSD, atrioventricular septal defect.

Table 2 Associated cardiac lesions in patients who underwent mitral valve replacement

\begin{tabular}{ll}
\hline Pathology & $\begin{array}{l}\text { Number of } \\
\text { patients }\end{array}$ \\
\hline Coarctation & 6 \\
Coarctation, ventricular septal defect & 2 \\
Coarctation, subaortic stenosis & 1 \\
Subaortic stenosis & 2 \\
Subaortic stenosis, pulmonary stenosis & 1 \\
Aortic stenosis & 2 \\
Pulmonary stenosis & 1 \\
Aortic regurgitation & 1 \\
Tricuspid regurgitation & 2 \\
Tetralogy of Fallot, left superior vena cava to & 1 \\
$\quad$ coronary sinus & \\
Large patent ductus arteriosus & 1 \\
Total & 20 \\
\hline
\end{tabular}

during the study period. Median (range) age at operation was 3.3 years ( 2 days to 18.1 years); 18 patients $(33 \%)$ were less than 2 years old. Median body weight was $12.1 \mathrm{~kg}$ (range $3.2-44.7 \mathrm{~kg}$ ).

Congenital mitral valve malformation was the most common underlying cardiac pathology $(72 \%$ of the patients), followed by endocarditis $(15 \%)$, stenosis of a prosthetic mitral valve placed before $1987(9 \%)$, and rheumatic valve disease $(4 \%)$ (table 1$)$. Associated cardiac defects were present in 37\% (table 2), and the majority (76\%) had undergone previous cardiac surgery. The indication for mitral valve replacement was mitral regurgitation in $63 \%$ of the cases, native or prosthetic mitral valve stenosis in $28 \%$, and mixed mitral valve disease in $9 \%$. During the study period five patients underwent mitral valve replacement twice because of prosthetic valve dysfunction.

SURGICAL TECHNIQUE

A median sternotomy was used in all but two patients who underwent a right thoracotomy. Operation was performed on cardiopulmonary bypass with moderate hypothermia and crystalloid cardioplegic arrest. In small infants a period of deep hypothermic circulatory arrest was used. The prosthesis was placed within the mitral annulus except in eight patients, in whom the valve was sutured in the supraannular position because the native valve ring did not admit the smallest available prosthesis. All patients received a mechanical valve. Seven

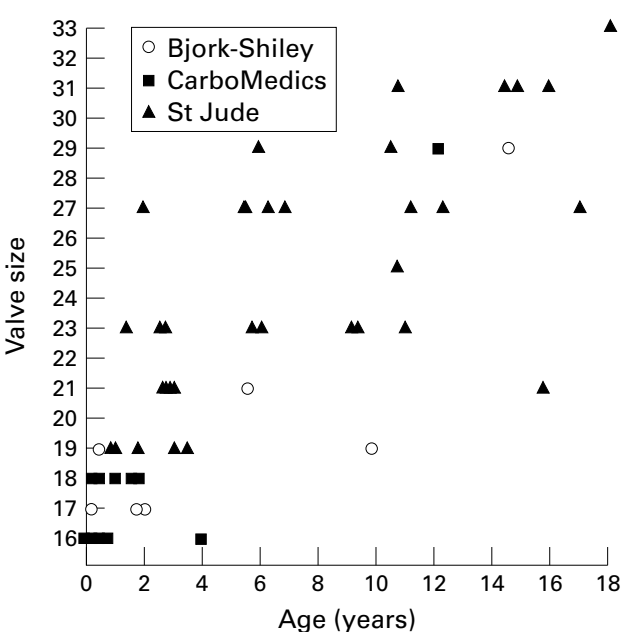

Figure 1 Age of the patient at the time of mitral valve replacement and size and type of prosthesis inserted. Younger children generally received smaller prostheses. Empty symbols, tilting disk prosthesis; filled symbols, bileaflet prosthesis.

Bjork-Shiley valves (Shiley Inc, Irvine, California, USA) were implanted until 1989, $38 \mathrm{St}$ Jude Medical prostheses (St Jude Medical Inc, St Paul, Minneapolis, USA) were used throughout the study period, and 14 CarboMedics valves (CarboMedics Inc, Austin, Texas, USA) were used since 1991. The distribution of valve size and age of the patients at operation is given in fig 1 . Concomitant cardiac surgical procedures were carried out in 11 patients. Three patients required implantation of a permanent pacemaker postoperatively for acquired atrioventricular block.

anticoagulation

Postoperatively all patients received oral anticoagulation with sodium warfarin, aiming at an international normalised ratio (INR) of between 2.5-3.5. Temporary anticoagulation with intravenous heparin was used during invasive or dental procedures, maintaining the partial thromboplastin time ratio between 1.5-2 times baseline. Antiplatelet drugs were not prescribed.

FOLLOW UP

Patients were seen at yearly intervals in the outpatient department, or more frequently if necessary. Information on patients living abroad was obtained by contacting the referring cardiologist. All surviving patients geographically available for follow up were invited for echocardiographic studies. The closing interval for data collection was from February to October 1998. Follow up information was complete for the immediate postoperative period and for all survivors resident in the UK. Four patients had moved abroad and could not be traced; therefore late follow up was $93 \%$ complete. Cumulative follow up for operative survivors was 205.4 years, with a median follow up of $4.1(0.2-11.3)$ years/patient.

ECHOCARDIOGRAPHIC STUDIES

All 30 operative survivors resident in the UK were invited to undergo an echocardiographic examination on a voluntary basis. One patient 
who was receiving palliative care for severe heart failure was too unwell to attend and one patient refused. The remaining 28 patients and one overseas patient who had returned for redo mitral valve replacement were studied at a median time of 6.6 years (14 days to 11.2 years) after their most recent mitral valve replacement. Twenty eight patients had a bileaflet valve and there was one tilting disk valve.

Transthoracic echocardiography was performed using commercially available echocardiography equipment (Acuson Sequoia, Acuson Co, Mountain View USA) with a $5 \mathrm{MHz}$ phased array transducer.

Left ventricular end systolic and end diastolic volumes were calculated from orthogonal apical views using a modified Simpson's rule and an on-line calculation package. Left ventricular systolic performance was then assessed by determination of the ejection fraction. The lack of normal landmarks in the left ventricle as well as the presence of paradoxical septal motion in some patients made estimation of the shortening fraction unreliable. The left ventricle was further evaluated for wall motion abnormalities and for outflow tract obstruction. The mitral valve prosthesis was studied from an apical position and peak inflow velocities of the $E$ and $A$ waves over the prosthesis determined. The inflow Doppler signals were digitised and the mean gradient across the valve computed. In addition a qualitative assessment was made of prosthetic valve leaflet motion and the presence of pannus or paravalvar leaks. The right atrioventricular valve was assessed for regurgitation and the right ventricular pressure estimated from the Doppler signal when possible.

DATA HANDLING

Morbidity and mortality following mitral valve replacement are reported according to published guidelines. ${ }^{14}$ The actuarial life method was used to describe survival and freedom from complications. Results are reported as an estimate of event-free survival with $70 \%$ confidence intervals (CI). The incidence of events at follow up is also reported as linearised rates expressed as percentage/patient/year (\% PPY). Risk factors for death within 30 days were examined using logistic regression. For evaluation of factors affecting overall survival and need for reoperation a Cox proportional hazards model was developed. Continuous data are presented as median with range. A probability value of $p<0.05$ was regarded as significant.

\section{Results}

MORTALITY AND MORBIDITY

Eleven patients $(20.3 \%)$ died within 30 days of operation. Nine of these were less than two years old. Most deaths occurred in the immediate postoperative period and were heart related (heart failure, 4; residual left ventricular outflow tract obstruction after resection of subaortic stenosis, 2; circumflex coronary artery injury, 1; pulmonary hypertension, 1). A further patient died in hospital because of ongoing sepsis, and there was one sudden death. One patient died at home from a cerebral event on the 26th postoperative day, presumably related to haemorrhage associated with over-anticoagulation.

Four patients required mechanical cardiac assistance immediately after operation. Two died of unrecoverable cardiac failure, but the two most recent cases were successfully weaned and are alive at 8 and 15 months of follow up, respectively. A further patient required mechanical support on the fourth postoperative day because of progressive deterioration of cardiac function. She was successfully weaned and discharged home but underwent heart transplantation for intractable heart failure four months after mitral valve replacement. She remains well four years later.

There were four late deaths. Two patients died in hospital from multiorgan failure after a protracted postoperative course at two and three months postoperatively, respectively. One patient died from chronic cardiac failure at eight months, and there was a sudden death at 13 months of follow up. Currently, two patients are in severe cardiac failure at 3.6 and 8.2 years of follow up, respectively. One is awaiting heart transplantation and the other has opted for palliative care.

Small prosthetic valve size and supraannular valve position were predictors of 30 day mortality $(p<0.05)$. No significant effect was demonstrated for year of operation, sequence number of operation, body weight, indication for operation, or concomitant surgical procedures. The actuarial probability of freedom from death, including 30 day mortality and transplantation, at 5 years of follow up was $68 \%(70 \% \mathrm{CI}, 62 \%$ to $75 \%)$. This was the same at 10 years (fig 2A). The linearised rate of late death was $1.9 \% \mathrm{PPY}$.

Significant bleeding complications during follow up occurred in six patients: following dental extraction (3), insertion of pacemaker box (1), haemarthrosis (1), and gastrointestinal bleed (1). The patients undergoing dental extractions were anticoagulated with intravenous heparin and the others received sodium warfarin. Anticoagulation was within reference limits in all patients except one, who suffered a spontaneous haemarthrosis with a raised INR of 4.5. All but one patient made a full recovery from their bleeding episode. The latter continues to suffer from hypochromic anaemia related to gastrointestinal blood loss. Minor nose and gum bleeds were often reported but were not included in the statistical analysis. One patient who suffered a fatal cerebral event on the 26th postoperative day, which was probably related to over-anticoagulation, was counted as a 30 day death and not as a bleeding complication. The estimated actuarial freedom from bleeding at 5 and 10 years was $89 \%$ (70\% CI $84 \%$ to $94 \%)$ and $65 \%(70 \%$ CI $50 \%$ to $80 \%$ ), respectively (fig $2 \mathrm{~B}$ ). The linearised event rate for bleeding was $2.9 \%$ PPY.

One thromboembolic episode was reported in a patient who suffered amaurosis fugax at nine months of follow up. The INR at the time of the event was 2.9. The actuarial probability for freedom from thromboembolism both at 5 
and 10 years was $97 \%(70 \%$ CI $95 \%$ to $99 \%)$. The linearised rate for thromboembolism was $0.5 \%$ PPY.

Postoperative endocarditis developed in two patients at two and eight years of follow up, respectively. On both occasions the infection (Streptococcus pneumoniae, 1; enterococcus, 1) was cured after six weeks of antibiotic treatment. The estimated actuarial freedom from postoperative endocarditis at five years was $96 \%(70 \%$ CI $93 \%$ to $99 \%)$ and at 10 years, $80 \%(70 \%$ CI $65 \%$ to $95 \%)$. The linearised rate was $1 \%$ PPY. Seven patients had a history of endocarditis before mitral valve replacement, including five with active bacterial infection at the time of surgery. Three patients, all with active infection, died within 30 days of surgery but there has been no recurrence of infection in the survivors.

Non-structural prosthetic valve dysfunction developed in five patients between 2.2 and 9.0 years of follow up (median 5.8). In four patients this reflected patient to prosthetic valve size mismatch as a result of somatic growth. In two of these patients there was also
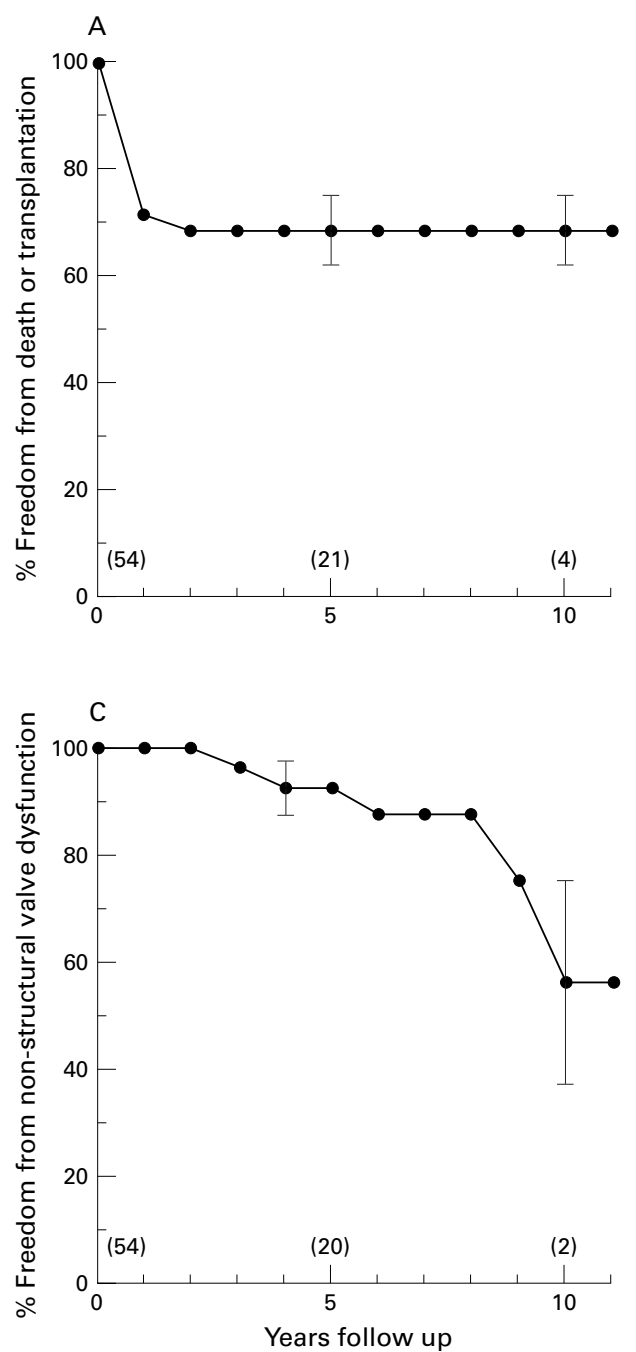

significant pannus formation at the ventricular aspect of the prosthesis. Successful redo mitral valve replacement with a larger prosthesis was performed in all cases, but one patient required a permanent pacemaker for postoperative heart block. A further case was a patient with a failed atrioventricular septal defect repair who underwent mitral valve replacement at the age of one year and developed significant left ventricular inflow and outflow obstruction from pannus formation and subaortic stenosis two years later. This patient underwent redo mitral valve replacement and resection of subaortic membrane but after a further two years of follow up has significant recurrent subaortic stenosis. Actuarial freedom from non-structural valve dysfunction at five years was $92 \%(70 \%$ CI $87 \%$ to $97 \%)$ and at 10 years, $56 \%(70 \%$ CI $37 \%$ to $75 \%$ ) (fig $2 \mathrm{C}$ ). The linearised rate was $2.4 \%$ PPY.

There was no valve thrombosis or structural valve failure. Redo operation was performed in five patients and was related to non-structural valve dysfunction in all cases.
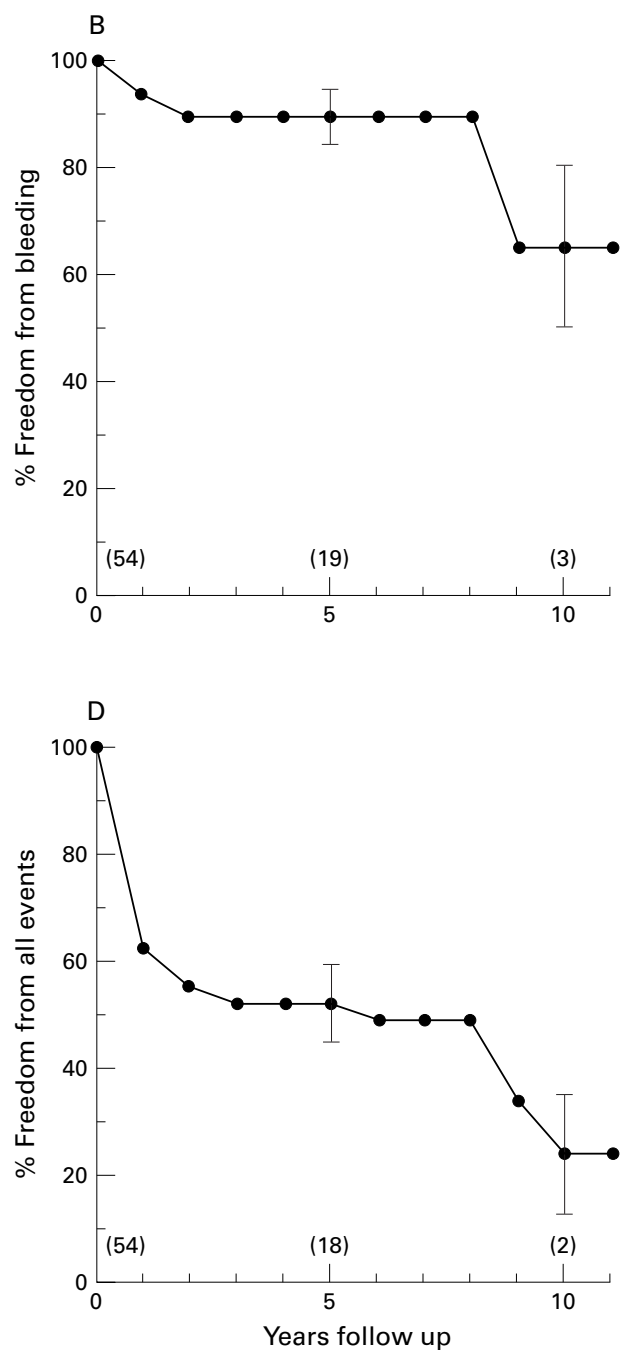

Figure 2 (A) Actuarial freedom from death or transplantation (including 30 day deaths) following mitral valve replacement; $70 \%$ confidence limits are indicated at 5 and 10 years. The numbers of patients originally at risk for the event at the time of the operation and at 5 and 10 years follow up are given in parentheses in this and all the following curves. These numbers become very small towards maximum follow up time. (B) Actuarial freedom from bleeding. (C) Actuarial freedom from non-structural valve dysfunction. (D) Event-free survival. 
The estimated event-free survival at 5 years and 10 years was $52 \%$ (70\% CI $45 \%$ to $60 \%)$, and $24 \%(70 \%$ CI $13 \%$ to $35 \%)$, respectively (fig 2D).

Multivariate analysis showed that low weight, young age at operation, and small prosthetic valve size increased the chance of death or reoperation $(p<0.05)$. Previous cardiac operation, sequence number of operation, concomitant surgical procedures, indication for operation, and supra-annular position were not associated with an increased risk.

ECHOCARDIOGRAPHIC STUDIES

Left ventricular end systolic dimension and end diastolic dimension normalised for body surface area were increased in a substantial number of patients (fig 3). Left ventricular ejection fraction varied widely (range $21-82 \%$, median $62 \%$ ). Wall motion abnormalities of the ventricular septum were documented in 12 patients $(41 \%)$ and in one case there was an obvious aneurysm of the left ventricular free wall. The estimated mean gradient over the mitral valve prosthesis was between 2.2 and $13.7 \mathrm{~mm} \mathrm{Hg}$ (median $6.6 \mathrm{~mm} \mathrm{Hg}$ ). Larger size valves were associated with a smaller mean gradient (Spearman rank correlation coefficient $-0.45, \mathrm{p}<0.05)$. There was no significant correlation between the duration of follow up and mean transvalvar gradient or ejection fraction. Measurement of right atrioventricular valve regurgitation, when present, suggested elevation of pulmonary artery pressures in patients with a high mean gradient over the mitral prosthesis. For those patients with the valve placed in a supra-annular position, there was a typical Doppler inflow signal. The E wave velocity was increased for a given mean gradient compared with patients in whom the valve had been placed in an annular position.

Subjective assessment of the mitral valve prosthesis revealed no signs of mechanical valve failure. The left atrium was free from thrombus in all cases. There was pannus formation on the ventricular side of the prosthesis in three patients causing obstruction to left ventricular inflow. Left ventricular outflow tract obstruction was documented in six $(21 \%)$. In two patients this was related to protrusion of the prosthesis into the outflow tract; in the other cases this was part of the underlying cardiac pathology. Significant paravalvar leaks were present in five patients, including one in which the jet extended into the right atrium. None of these patients was clinically symptomatic or suffered haemolysis.

\section{Discussion}

To our knowledge this is one of the largest studies on mitral valve replacement in children with mechanical prostheses that are currently in clinical use. There are several findings that stand out and need further discussion-the high 30 day mortality, the bleeding complications, and redo mitral valve replacement for somatic growth.

Mitral valve replacement carried a 30 day mortality of $20.3 \%$ and this was associated with small prosthetic valve size and supra-
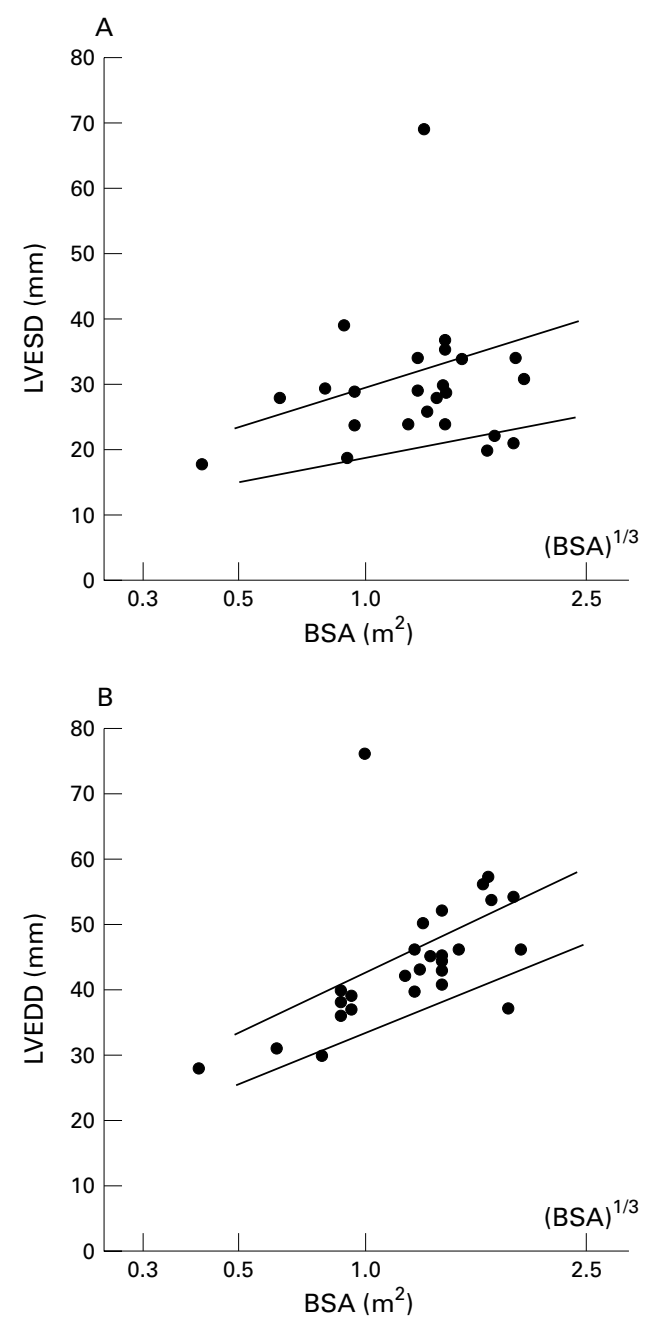

Figure 3 Echocardiographic measurement of left ventricular internal diameter according to body surface area. Reference lines indicate $95 \%$ prediction limits. $(A)$ Measurement at end systole. (B) Measurement at end diastole. $B S A$, body surface area; $L V E D D$, left ventricular end diastolic dimension; LVESD, left ventricular end systolic dimension.

annular position. Other investigators have also reported a high operative mortality. ${ }^{7-10}$ Mitral stenosis has previously been identified as a risk factor, ${ }^{9}{ }^{10}$ but this was not confirmed in our series. Placement of the valve in the supraannular position was related to poor outcome in some studies, ${ }^{15}{ }^{16}$ but not in others. ${ }^{11}$ In our series, valves placed in a supra-annular position produced noticeably different Doppler inflow characteristics, possibly related to decreased left atrial size and compliance. Such a finding may predispose to pulmonary hypertensive episodes and therefore might contribute to the poor outcome in some patients with valves placed in a supra-annular position.

Mechanical cardiac assistance was successfully used to overcome severe postoperative cardiac failure in three of five cases. A left ventricular assist device was used once in the early part of the experience and extracorporeal membrane oxygenation (ECMO) in all other cases. The first two patients who received mechanical cardiac assistance died within one and four days. Both patients had undergone mitral valve replacement for severe left atriov- 
entricular valve regurgitation after a previous repair of an ostium primum septal defect. A third patient was put on ECMO for progressive deterioration of cardiac function on postoperative day 4 and was successfully weaned four days later. More recently ECMO was used in two patients who underwent mitral valve replacement for acute mitral regurgitation related to bacterial endocarditis. These patients were successfully weaned on days 4 and 8 , respectively, and there has been no recurrence of endocarditis. The recent encouraging results may be related to better patient selection and our increasing experience with extracorporeal support gained through our ECMO programme.

We use sodium warfarin to achieve anticoagulation after mitral valve replacement, aiming at an INR of between 2.5-3.5. This regimen resulted in a very low incidence of thromboembolism and no valve thrombosis, but apparently at the cost of a significant number of bleeding events. However, four bleeding episodes were related to surgical interventions and one was related to overanticoagulation; thus the number of spontaneous bleeding complications in active children anticoagulated within reference limits was very low.

There may still be a case, nonetheless, to reduce the level of anticoagulation in our patients. Technical improvements in valve design are thought to have greatly reduced the thrombogenic potential of the currently used bileaflet prostheses compared with previous generations of mechanical valves. ${ }^{17}$ Unfortunately, this has not been uniformly confirmed in long term follow up studies in large populations of adult patients, ${ }^{18}{ }^{19}$ suggesting that the specific characteristics of patients may play a greater role in thromboembolism than the type of mechanical prosthesis used. The high intrinsic heart rate and preservation of sinus rhythm in children are thought to protect against thrombosis, and indeed in our echo studies no intracardiac clot was identified. Support for the low thrombogenic potential of modern bileaflet valves in children is also provided by Ibrahim and colleagues. ${ }^{20}$ They reported no thromboembolic or bleeding complications in a small group of patients with St Jude Medical prostheses anticoagulated with an INR of between 2.0-2.5. Duran and colleagues found to their surprise that there were no cases of thromboembolism in a poorly compliant population of children with CarboMedics valves, despite the fact the nearly half the patients failed to reach the target prothrombin time of 1.5 to 2 times baseline. ${ }^{21}$ However, there was one case of mitral valve thrombosis in a patient on antiplatelet treatment. Finally, in a recent prospective study comparing coumadin with a combination of aspirin plus dipyridamole, Bradley and co-workers found no statistical difference in rates of thromboembolism and bleeding. ${ }^{22}$ The level of anticoagulation and the role of antiplatelet treatment for prevention of thromboembolic complications after mechanical valve replacement in children remains controversial. Because of the catastrophic effect of most thromboembolic complications it remains policy in our unit to continue anticoagulation with sodium warfarin.

The use of small prosthetic valves in young children will ultimately result in patient to prosthetic valve mismatch and restriction of haemodynamic function. It seems justified, therefore, to place the largest possible prosthesis at the time of operation, but caution is warranted as insertion of a tight fitting valve in the mitral annulus in two infants in our series led to circumflex coronary artery injury and profound cardiac failure.

It is to be expected that redo mitral valve replacement for somatic growth will eventually become necessary in a substantial number of patients. Little is known about the characteristics of these patients or the timing of redo surgery, and it is therefore useful to examine the eight patients who had a further mitral valve replacement for this reason in our series in more detail. Five patients who entered the study had redo mitral valve replacement for somatic growth (table 1) and a further four redo operations were performed during the follow up period, including one in a patient who had outgrown his second mitral prosthesis. The age at first mitral valve replacement varied widely (median 4.5 years, range 1 month to 10 years), as did the time interval between valve replacements (median 6.8 years, range 3.4-9.6 years). The decision for redo mitral valve replacement was based on a combination of clinical symptoms and haemodynamic studies showing an increased transvalvar gradient with evidence of raised pulmonary artery pressures, but haemodynamic findings and symptoms were generally poorly correlated. Redo operation was carried out as an elective procedure in all except one patient, who underwent urgent surgery because of heart failure related to severe restriction of movement of a BjorkShiley valve. All patients survived the operation and, in keeping with earlier reports, ${ }^{10}{ }^{12}$ a larger prosthesis could be placed on nearly all occasions (fig 4). Furthermore, in many cases a bileaflet valve was inserted, which offers improved effective orifice area and better flow dynamics for the same external diameter as

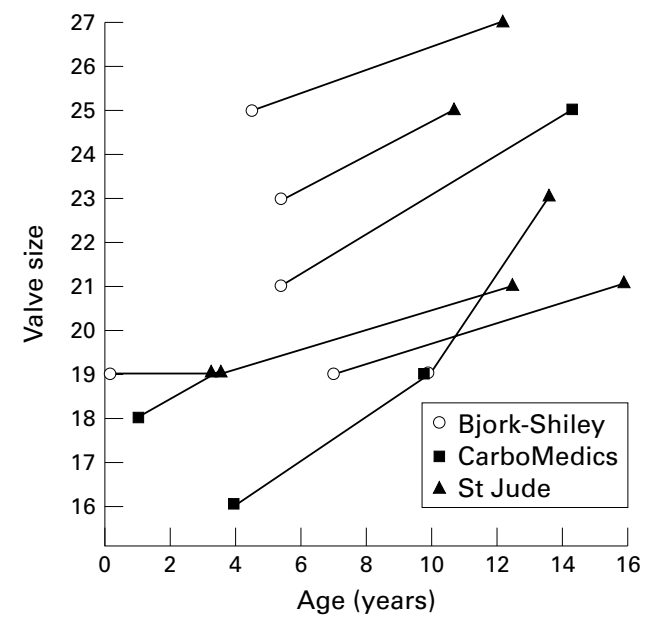

Figure 4 Increment in prosthetic valve size achieved in children who underwent redo mitral valve replacement. 
earlier generation tilting disk valves. Removal of pannus also contributed to relief of obstruction. Postoperative evaluation in some patients, however, revealed a higher than expected gradient across the new valve, suggesting that factors other than valve size contribute to the left ventricular inflow obstruction.

We were concerned by the echocardiographic finding that the left ventricular dimensions were either above normal or towards the upper limit of normal in several of the patients. Wall motion abnormalities were also common, especially in those patients in whom the mitral subvalvar apparatus had been excised at the time of mitral valve replacement, indicating its importance in maintaining normal ventricular geometry. The absence of a positive statistical correlation between ventricular dimensions and length of follow up, however, may suggest that there is no progressive deterioration in ventricular volumes over time, even in those patients in whom the subvalvar support has been removed. Although we now aim to preserve the posterior leaflet with supporting chordal apparatus at the time of mitral valve replacement, this has only been possible in a few cases. In many patients with congenital mitral valve disease, the structure of the subvalvar tissue is abnormal and may contribute to mitral valve obstruction. Resection of this tissue at the time of valve replacement is then necessary to achieve unobstructed inflow to the left ventricle. Resection may further be required to create space for the insertion of an adequately sized valve prosthesis. The latter is particularly true for young children if early patient/prosthetic mismatch is to be avoided.

\section{STUDY LIMITATIONS}

This study had several limitations including the size and heterogeneity of the study population. Moreover, the retrospective design of the study has the associated problems of incomplete data collection. Although these limitations make it difficult to draw many firm conclusions, it should not be forgotten that mitral valve replacement in children is uncommon and therefore single centre experience will involve only limited numbers of patients. A European study is currently under way with the aim of overcoming some of the limitations related to small patient numbers.

CONCLUSIONS

Mitral valve replacement in children remains a challenge. With the use of mechanical prostheses the problem of structural valve failure is not an issue any more. However, the procedure is still associated with a high complication rate, both at operation and at follow up, and should therefore be reserved for patients in whom mitral valve repair is not possible.

We are grateful for the support of Adelaide Tunstill, clinical nurse specialist, whose experience and personal knowledge of the patients was invaluable for achieving the extensive follow up in this series. We thank the patients and their parents for participating in this study.

1 Davachi F, Moller JH, Edwards JE. Diseases of the mitral alve in infancy. An anatomic analysis of 55 cases. Circulaion 1971;43:565-79.

2 Collins-Nakai RL, Rosenthal A, Castenada A, et al. Congenital mitral stenosis: a review of 20 years' experience. Circulation 1977;56:1039-47.

3 Carpentier A, Branchini B, Cour JC, et al. Congenital malformations of the mitral valve in children: pathology and surgical treatment. $\mathcal{F}$ Thorac Cardiovasc Surg 1976;72:85466.

4 Stellin G, Bortolotti U, Mazzucco A, et al. Repair of congenitally malformed mitral valve in children. F Thorac Cardiovasc Surg 1988;95:480-5.

5 Sousa Uva M, Galletti L, Lacour Gayet F, et al. Surgery for congenital mitral valve disease in the first year of life. $\mathcal{F}$ Thorac Cardiovasc Surg 1995; 109:164-76.

6 Thorac Cardiovasc Surg 1995,109.164-76. Dunn JM. Pocine valve
Surg 1981;32:357-68.

7 Cohn LH, Reut RM. Mechanical and bioprosthetic mitral valve replacement. In: Edmund LH Jnr, ed. Cardiac surgery in the adult. New York: McGraw-Hill, 1997:1040-3.

8 Milano A, Vouhe PR, Baillot-Vernant F, et al. Late results after left-sided cardiac valve replacement in children. $\mathcal{F}$ Thorac Cardiovasc Surg 1986;92:218-25.

9 Almeida RS, Elliott MJ, Robinson PJ, et al. Surgery for congenital abnormalities of the mitral valve at the Hospital for Sick Children, London from 1969-1983. 7 Cardiovasc Surg 1988;29:95-9.

10 Zweng TN, Bluett MK, Mosca R, et al. Mitral valve replacement in the first 5 years of life. Ann Thorac Surg 1989;47:720-4.

11 Kadoba K, Jonas RA, Mayer JE, et al. Mitral valve replacement in the first year of life. F Thorac Cardiovasc Surg 1990; 100:762-8.

12 Nudelman I, Schachner A, Levy MJ. Repeated mitral valve replacement in the growing child with congenital mitra valve disease. F Thorac Cardiovasc Surg 1980;79:765-9.

13 van Doorn C, Yates R, Tunstill A, et al. Quality of life in children following mitral valve replacement. Heart 2000;84: 643-7.

14 Edmunds LH Jnr, Clark RE, Cohn LH, et al. Guidelines for reporting morbidity and mortality after cardiac valvular operations. Ann Thorac Surg 1996;62:932-5.

15 Schaffer MS, Clarke DR, Campbell DN, et al. The St Jude Medical cardiac valve in infants and children: role of anticoagulant treatment. F Am Coll Cardiol 1987;9:235-9.

16 Adatia I, Moore PM, Jonas RA, et al. Clinical course and hemodynamic observations after supraannular mitral valve replacement in infants and children. $\mathcal{F}$ Am Coll Cardiol 1997;29:1089-94.

17 Butchart EG. Prosthesis-specific and patient-specific anticoagulation. In: Butchart EG, Bodnar E, eds. Thrombosis, embolism and bleeding. London: ICR Publishers, 1991:239317.

18 Horstkotte D, Schulte HD, Bircks W, et al. Lower intensity anticoagulation treatment results in lower complication rates with the St Jude Medical prosthesis. F Thorac Cardiovasc Surg 1994;107:1136-45.

19 Fiore AC, Naunheim KS, D’Orazio S, et al. Mitral valve replacement: randomized trial of St Jude and Medtronic Hall prostheses. Ann Thorac Surg 1992;54:68-73.

20 Ibrahim M, Cleland J, O'Kane H, et al. St Jude Medical prosthesis in children. 7 Thorac Cardiovasc Surg 1994;108: $52-6$.

21 Duran CMG, Gometza B, Martin-Duran R, et al. Performance of 96 CarboMedics valve replacements in 75 patients less than twenty-one years of age. Ann Thorac Surg 1994;58:639-45.

22 Bradley SM, Sade RM, Crawford FA Jnr, et al. Anticoagulation in children with mechanical valve prostheses. Ann Thorac Surg 1997;64:30-6. 